\title{
Analysis of $A E$ characteristics of tool wear in drilling CFRP/Ti stacked material
}

\author{
Zhan Wang ${ }^{1}$, Sheng Leng ${ }^{2}$, Tao $\mathrm{Min}^{3}$, and Gang $\mathrm{Chen}^{4}$ \\ ${ }^{1,2,3}$ College of Mechanical and Electrical Engineering, Nanjing University of Aeronautics and \\ Astronautics29\# Yudao Street, Nanjing, Jiangsu 210016, China \\ ${ }^{4}$ AVIC Changhe Aircraft Industry (Group) Co., Ltd., Jingdezhen City, Jiangxi, 333002, China \\ wz72580@nuaa.edu.cn;meesleng@nuaa.edu.cn;lymintao@foxmail.com;13707984109@163.com
}

\begin{abstract}
Based on the signal data acquired in drilling process of carbon fiber reinforced polymer/titanium alloy (CFRP/Ti) stacked materials, the acoustic emission (AE) characteristic values were carefully studied, by using the method of statistical analysis, spectrum analysis and wavelet packet. The results show that the root mean square(RMS) value of the $\mathrm{AE}$ signals and the energy of the wavelet packet are closely related to the tool wear. Meanwhile, experiments indicate that different materials, chips and tool tipping will cause instantaneous signal mutation, which has different forms in time domain and in time-frequency domain. These mutations may increase the difficulty of identifying the tool wear. Fortunately, with repeated experiments and comparison, some identifiable mutations were recognized. When a tool is processed from CFRP to $\mathrm{Ti}$, the signal intensity decreases generally, the high-frequency component of signal increases gradually, and the signal has a tendency to show in high frequencies.
\end{abstract}

\section{Introduction}

Composite stacked material is widely used in many fileds, such as aircraft, rocket, missile and so on. In order to improve the positional accuracy of the assembly hole, the method of one-off drilling the stacked material is widely applied to the machining of holes. There are great differences in cutting performance because of the difference of materials. Therefore, the quality of holes is difficult to guarantee, and tool wear is serious. The tool test must be recarried out to determine the tool life when the processing conditions change. As a result, the tool test can ensure the quality of the holes, but it will cause the cutting tool to be scrap in advance and the reduction of production efficiency.

AE signal is elastic stress wave signal, which produced by the sudden release of elastic deformation in the material, and it is widely used in non-destructive monitoring now. So far, researchers at home and abroad have applied AE method to tool wear monitoring such as turning, milling and drilling. From their studies, some effective results also have been obtained. For example, K Jemielniak had studied that the kurtosis and energy of AE signal are sensitive to the tool wear condition, [1]. Neslušan used two AE sensors to collect high and low frequency of AE signal, and the ratio of two signals was selected as characteristic parameter to evaluate the tool wear, [2]. In Xie Jianfeng's research, the AE signal [31.25$250] \mathrm{KHz}$ frequency energy ratio was used as the feature of tool wear monitoring,[3]. Zhang 
dongliang proved that the tool wear can be indicated effictively by embedding dimension and Lyapunov coefficient, [4]. Gómez M P studied that the mean energy of AE signal is closely related to tool wear and it can be used to monitor tool wear, [5]. Hu Jianglin studied that the speed of tool wear can be expressed clearly by the number of AE signal ringing,[6].

\section{Design and analysis of experiments}

The studies above show that AE signals can be used as tool monitoring effectively, [7]. At present, the study on features of AE signal when put drilling processing on CFRP/Ti structure has not been reported yet. So, based on the signal data obtained from drilling process of CFRP/Ti materials, the AE characteristic values such as the means, skewness, kurtosis, root mean square and wavelet packet energy were carefully studied in this paper.

The experimental material is CFRP/Ti, and the thickness of CFRP plate and Ti plate is $3 \mathrm{~mm}$ respectively. The type of data acquisition card is Advantech PCIE1816H, whose sample rate is $1 \mathrm{MS} / \mathrm{s}$, and the frequency range of the target $\mathrm{AE}$ signal is $125 \mathrm{KHz}-500 \mathrm{KHz}$. The model of sensor is Shenghua SR800. The range of working frequency of the sensor is $50 \mathrm{KHz}-800 \mathrm{KHz}$, and the peak of sensitivity is greater than $70 \mathrm{db}$. In the experiment, the sensor was mounted on the surface of the CFRP plate. At the same time, coupling agent is used on the surface between the sensor and CFRP plate to reduce the loss of signal. The hardware platform for collecting signals is shown in Figure 1.

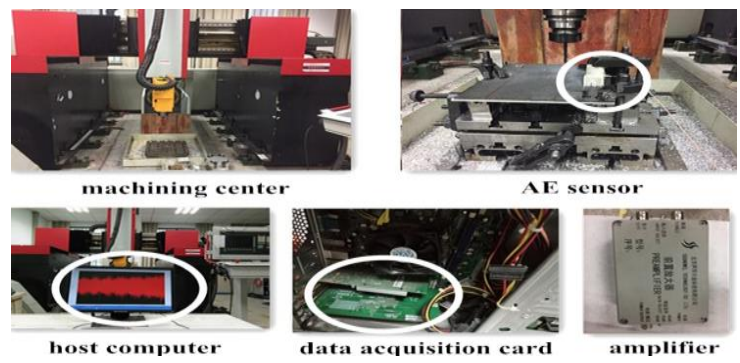

Fig. 1. Signal acquisition hardware settings.

For the convenience of research, the CFRP layer was identified as F, and the Ti layer was identified as L. The drill can cause a signal mutation when it is just in touch with the workpiece or rapid return. In this paper, only stable signals in $\mathrm{F}$ and $\mathrm{L}$ will be analysed.

\subsection{Experiment and analysis of high speed steel drill}

The drilling experiment was carried out firstly with the easy to wear high speed steel bit. An uncoated high speed steel drill with $6 \mathrm{~mm}$ diameter was used in experiments, every tool is processed from new to worn. Experimental parameters are shown in Table 1.

Table 1. Experimental parameters of high speed steel drill.

\begin{tabular}{|c|c|c|c|c|}
\hline Groups No. & $\begin{array}{c}\text { Spindle speed } \\
\mathrm{n}(\mathrm{r} / \mathrm{min})\end{array}$ & $\begin{array}{c}\text { Tool feed } \\
\mathrm{f}(\mathrm{mm} / \mathrm{min})\end{array}$ & $\begin{array}{c}\text { Repetitions } \\
(\mathrm{N})\end{array}$ & $\begin{array}{c}\text { Number of } \\
\text { holes }\end{array}$ \\
\hline 1 & 3500 & 70 & 2 & 6 \\
\hline 2 & 3500 & 50 & 2 & 6 \\
\hline 3 & 2700 & 70 & 2 & 6 \\
\hline 4 & 2700 & 50 & 2 & 6 \\
\hline
\end{tabular}


Frequency domain analysis: We used high-pass digital filter to filter out the original signals below 100KHz. Meanwhile, Pwelch function was used to analyse the filtered signal, and the method of Hanning window was applied to the analysis of power spectrum. As a result, there are two states about the power spectrum of AE signal in the wear process of every high speed steel drill. We can find that the signal's power spectrum marked from 1 to 3 are maintained at one level, and the value of hole 4 reduced to another level abruptly, while the hole 5 and 6 are maintained at the same level with the hole 4. Especially, the rules are more obvious when the spindle speed belows 3500r/min. So, it can be concluded that the power spectrum value of AE signal will decrease when the tool wear is serious.

Time domain analysis: Similarly, filter out the signals below $100 \mathrm{KHz}$ in the original signals. Then, the characteristic values of mean, root mean square (RMS) value, skewness and kurtosis were calculated. It is found that the RMS value has a periodical characteristic. But, the mean, skewness and kurtosis have no obvious regularity. As shown in Figure 2, the variation of RMS value in $\mathrm{F}$ and $\mathrm{L}$ is in accordance with the change law of power spectrum.

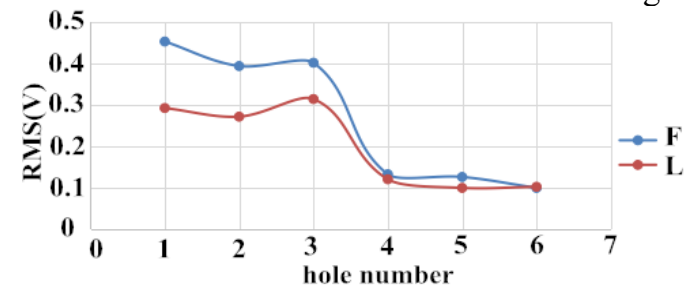

Fig. 2. Changes of RMS value with hole number in $\mathrm{F}$ and $\mathrm{L}$.

\subsection{Experiment and analysis of carbide drill}

The study above shows that there is a significant relationship between the power spectrum, RMS value of the AE signal and the tool wear condition . Obviously, it is hard to achieve the detailed characteristics of AE signals because the wear process of high speed steel drill is very fast. Therefore, the second stage experiments was carried out under the same conditions using the carbide drill, and the experimental parameters are shown in Table 2.

Table 2. Experimental parameters of carbide drill.

\begin{tabular}{|c|c|c|c|c|}
\hline Groups No. & $\begin{array}{c}\text { Spindle speed } \\
\mathrm{n}(\mathrm{r} / \mathrm{min})\end{array}$ & $\begin{array}{c}\text { Tool feed } \\
\mathrm{f}(\mathrm{mm} / \mathrm{min})\end{array}$ & $\begin{array}{c}\text { Repetitions } \\
(\mathrm{N})\end{array}$ & $\begin{array}{c}\text { Number of } \\
\text { holes }\end{array}$ \\
\hline 1 & 3500 & 70 & 2 & 26 \\
\hline 2 & 3500 & 50 & 2 & 26 \\
\hline 3 & 2700 & 70 & 2 & 26 \\
\hline 4 & 2700 & 50 & 2 & 26 \\
\hline
\end{tabular}

Frequency domain analysis: The power spectrum analysis of signals in $\mathrm{F}$ and $\mathrm{L}$ shows that the AE signal frequency band from $300 \mathrm{KHz}$ to $400 \mathrm{KHz}$ is regular. Specifically, from hole 1 to hole 7, the power spectrum value continues to rise. However, the power spectrum value of hole 8 , hole 9 and hole 10 continues to decline. Besides, from hole 11 to hole 26, the power spectrum value is stable. Comparing to the high speed steel bit, the signal power spectrum of carbide drill from the new to the wear process also can be divided into two states. But, the first state of the carbide drill presents a slow rising trend, and there is a short transition process from the first state to the second state. 
Time domain analysis: Filter out the original signals below $100 \mathrm{KHz}$. Then, the RMS value will be calculated. It is found that the regulation of signal RMS value is consistent with the power spectrum value. That is to say, in the initial wear stage, the RMS value of the signal rises steadily. After entering the stable wear period, the RMS value of the signal is stable near a certain level. The regulation of RMS value is shown in Figure 3.

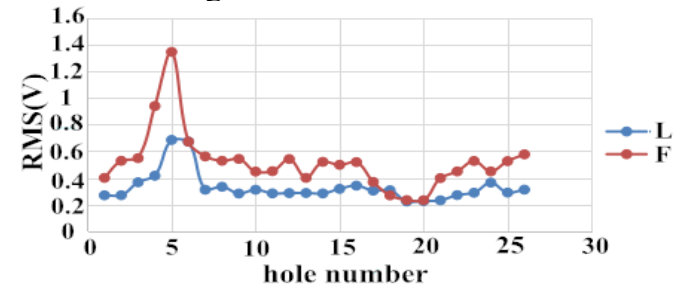

Fig. 3. Changes of RMS value with hole number in F and L.

From above analysis, it can be obtained that the power spectrum and the RMS value show a phased feature along with the tool wear, but the wear figure can not be indicated. So, in next paper, the method of wavelet packet transform will be applied to the analysis of $\mathrm{AE}$ signal, and the AE signal energy in each frequency section will be studied too.

\subsection{Wavelet packet analysis}

The wavelet packet analysis method ${ }^{[8]}$ has excellent resolution in low frequency and high frequency parts of the signal. The decomposition algorithm is as follows:

$$
\left\{\begin{array}{l}
x_{j+1,2 n}=\sum_{k} h_{0}(k-2 l) x_{j, n, k} \\
x_{j+1,2 n+1}=\sum_{k} h_{1}(k-2 l) x_{j, n, k}
\end{array}\right.
$$

In the formula, $\left\{x_{j, n, k}\right\}$ is the previous decomposition result of wavelet packet, $\left\{x_{j+1,2 n}\right\}$ and $\left\{x_{j+1,2 n+1}\right\}$ are the next decomposition results. Meanwhile, the scale index is $j$, the positioning index is $l$, the frequency index is $n$, the variable is $k$, the multiresolution filter coefficients using to record decomposition results are $h_{0}$ and $h_{1}$.

The wavelet packet reconstruction algorithm is as follows:

$$
x_{j, n, k}=2\left\lfloor\sum_{l} h_{0}(k-2 l) x_{j+1,2 n+1, k}+\sum_{l} h_{1}(k-2 l) x_{j+1,2 n, k}\right\rfloor
$$

According to formula (1) and (2), in time domain, there is a equality between the energy of original signal and the decomposition coefficient of wavelet packet. That is to say:

$$
E_{j, n}=\sum_{k=1}^{N}\left|x_{j, n, k}\right|^{2}
$$

\subsection{Analysis results}

Based on the above, the original signals of $\mathrm{F}$ and $\mathrm{L}$ were decomposed into three-layer, using the method of wavelet $\mathrm{db} 3$ analysis, and there are eight sections. Besides, the sample frequency is $1 \mathrm{MS} / \mathrm{s}$ and the width of each section is $62.5 \mathrm{kHz}$. Necessarily, we ignored the worthless signals in the range from 0 to $125 \mathrm{KHz}$ and 187.5 to $250 \mathrm{KHz}$. Then, the signals were reconstructed with the remaining frequency sections, and we decomposed the 
reconstructed signals into four-layer. So, the percentage of the wavelet packet energy in each frequency section was calculated. The result shows that there are four sections such as $19([125-156.25] \mathrm{KHz}), \quad 21([187.5-218.75] \mathrm{KHz}), \quad 22([218.75-250] \mathrm{KHz}) \quad$ and $27([375-$ $406.25] \mathrm{KHz}$ ) in $\mathrm{F}$, where the signals changed regularly with the tool wear. Furthermore, with the wear of the tool, the energy ratio of wavelet packet is gradually decreasing in the 21 and 22 sections of F, while the energy ratio of wavelet packet in the 19 and 27 sections is increasing gradually. The law of F also applies to L. However, in L, the signals were affected by the chip of $\mathrm{Ti}$, and the AE signal fluctuates greatly. The law of energy ratio is shown in Figure 4 and Figure 5. In Figure 4, the energy ratio of 19 and 27 sections can be divided into $\mathrm{AB}, \mathrm{CD}$ and $\mathrm{EF}$. The holes in $\mathrm{AB}$ segment are of good quality, and the holes of CD segment begin to appear slight burrs, while the holes of EF segment can be seen to have appeared obvious burrs. Besides, the quality of holes in each stage is shown in Figure 6.

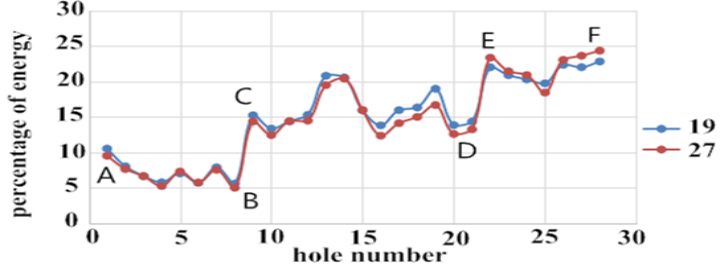

Fig. 4. Variation of energy percentage at 19 and 27 in F.

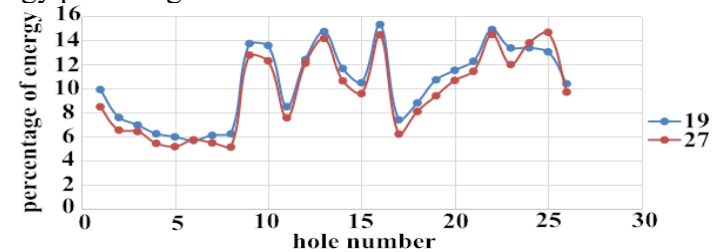

Fig. 5. Variation of energy percentage at 19 and 27 in L.

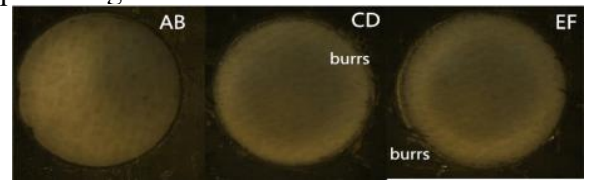

Fig. 6. Hole quality of each stage.

\subsection{Signal analysis in the area of contact surface}

As a result, the rule of contact surface characteristics in time domain are shown in Figure 7.

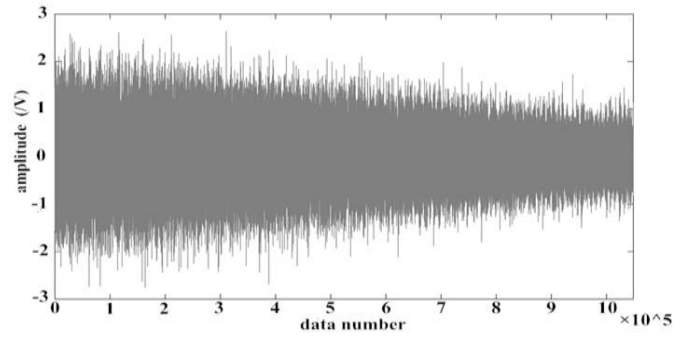

Fig. 7. Variation of amplitude in contact surface.

When the tool was processed from CFRP to Ti, the amplitude of the signal decreases gradually. Next, the method of wavelet packet multi-resolution is used to analyze the signal components of each frequency section. Also, it can be discovered that the signal energy is mainly concentrated in the 19,20,21,22 and 27 sections. Besides, the energy ratio of some main frequency sections is shown in Figure 8. The energy ratio of section 22 has a 
downward trend, and the frequency section 27 is on the rise as a whole. However, the energy ratio of the frequency section 20 is not sensitive to the tool wear.

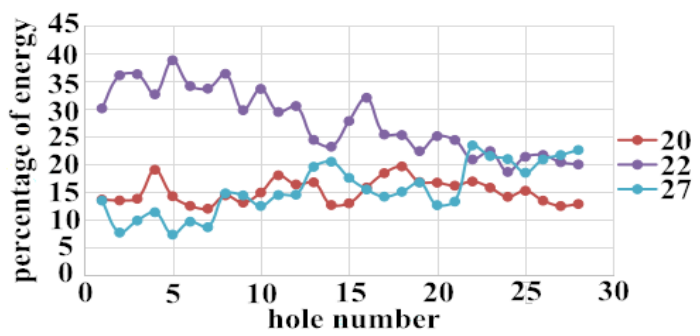

Fig. 8. Variation of energy percentage in some main frequency sections.

\section{Conclusions}

1)In the experiments of high speed steel drill, we find that the signal energy is mainly concentrated from $125 \mathrm{KHz}$ to $250 \mathrm{KHz}$ frequency range. The signal's RMS value produced by a high speed steel tool is at a high level in the initial stage, which indicated that the signal has a strong energy at the beginning. Besides, the initial energy produced by a carbide drill is low and it will increase gradually. So, we can achieve that the RMS value of the AE signals is closely related to the tool wear.

2)In the machining process of carbide drill, the rule of AE signal in CFRP layer is obvious with the tool wear. As a result, with the wear of tool, the energy ratio of signal in frequency section $19([125-156.25] \mathrm{KHz})$ and $27([375-406.25] \mathrm{KHz})$ increases, while it decreases in frequency section $21([187.5-218.75] \mathrm{KHz})$ and $22([218.75-250] \mathrm{KHz})$.

3)Different materials, chip and tool tipping will cause instantaneous signal mutation, which is shown in the form of an obvious spikes in the signal waveform in time domain. Furthermore, at a certain moment, the mutation is expressed in the form of the change of the signal components in the time-frequency domain. When a tool is processed from CFRP to $\mathrm{Ti}$, the signal intensity decreases and the high-frequency component of signal increases gradually, and the signal has a tendency to show in high frequencies. However, there still exists some problems, including diagnosing tool collapse and the model of tool wear prediction in CFRP/Ti structure, which have not yet been identified in this paper and need to be solved in future.

This work is funded by the National Natural Science Foundation, China (No. 51205201)

\section{References}

[1] Jemielniak K, Kossakowska J, Urbański T, P I Mech Eng B-J Eng 1(-1), 1-7 (2011)

[2] M. Neslušan, B. Mičieta, A. Mičietová, et al, Measurement 70, 1-13 (2015)

[3] Xie Jianfeng, Wang Haili, Modular Machine Tool \& Automatic Manufacturing Technique (5), 14-17 (2011)

[4] Zhang Dongliang, Mo Rong, Comput Integr Manuf 21(8), 2138-2146 (2015)

[5] Gómez M P, Hey A M, Ruzzante J E, et al, Phys. Procedia 3(1), 819-825 (2010)

[6] Hu Jianglin, Zhang Shaowen, Li Liang, Tool Engineering 46(3), 67-69 (2012)

[7] Liang S Y, Dornfeld D A, J. Eng. Indus. 111(3), 147-149 (1989)

[8] Nie Peng, Chen Xin, Journal of Beijing University of Aeronautics and Astronautics 37(1), 106-109 (2011) 